\title{
Learning In Neural Models With Complex Dynamics
}

\author{
Michael Stiber $^{*}$ and José P. Segundo ${ }^{\dagger}$
}

Technical Report HKUST-CS93-7

April 1993

${ }^{*}$ Department of Computer Science

The Hong Kong University of Science and Technology

Clear Water Bay, Kowloon, Hong Kong

${ }^{\dagger}$ Department of Anatomy and Cell Biology

and Brain Research Institute

University of California

Los Angeles, California 90024, USA

\footnotetext{
$\ddagger$ This work was supported by the Hong Kong Research Grants Council (DAG92/93.EG18), Trent H. Wells, Jr. Inc., and by a grant of computer time made possible under a joint study with the IBM Corporation on the IBM 3090/600J Supercomputer at the UCLA OfÆcef Academic Computing.
} 


\begin{abstract}
Interest in the ANN Æellas recently focused on dynamical neural networks for performing temporal operations, as more realistic models of biological information processing, and to extend ANN learning techniques. While this represents a step towards realism, it is important to note that individual neurons are complex dynamical systems, interacting through nonlinear, nonmonotonic connections. The result is that the ANN concept of learning, even when applied to a single synaptic connection, is a nontrivial subject.

Based on recent results from living and simulated neurons, a Ærqtass is made at clarifying this problem. We summarize how synaptic changes in a 2-neuron, single synapse neural network can change system behavior and how this constrains the type of modiÆcatioscheme that one might want to use for realistic neuron-like processors.
\end{abstract}




\section{Introduction}

Biological nervous systems are networks of synaptically-coupled neurons, each behaving as a dynamical system producing short-lived voltage spikes. The input/output behavior of each neuron can be thought of as the transformation of trains of input spikes, via synapses, into trains of output spikes. This synaptic coding can be thought of as the operational unit of nervous systems [1]. Therefore, understanding synapses is a necessary, though not sufÆcien\$tep towards understanding neural computation.

When we look at the different types of synaptic coding seen in biological systems, we face at once the need to speak in terms of the behaviors of individual neurons, whether in isolation or in response to some input. These behaviors had been noted in a general sense quite some time ago [2], and have also been the subject of more recent work which, using techniques from the Æeld of nonlinear dynamics, has shed a great deal of light on their detailed characteristics $[3,4,5]$. One clear thing is that the neuron is a complex entity whose synaptic coding is anything but the simple, monotonic relationship embodied by the typical weighted-summation models used in ArtiÆciaXeural Networks.

Given this information, we might naturally question how one of the foci of ANN work, namely learning, can be related to the actual biological system. Here, learning is considered to involve the systematic, purposeful modiÆcationf neural behavior. If ANN learning is analogous to changing the response characteristics of a synapse (for instance, changing its strength by changing its maximum ionic permeability), then how does changing the synapse change the neuron's behavior? In other words, as we modify synaptic strength, how does the synaptic coding change? How might this behavioral change be useful in terms of changing the computation of the overall network? And, based on this information, what constraints might we place on any scheme for synapse-level TMlearning"? 


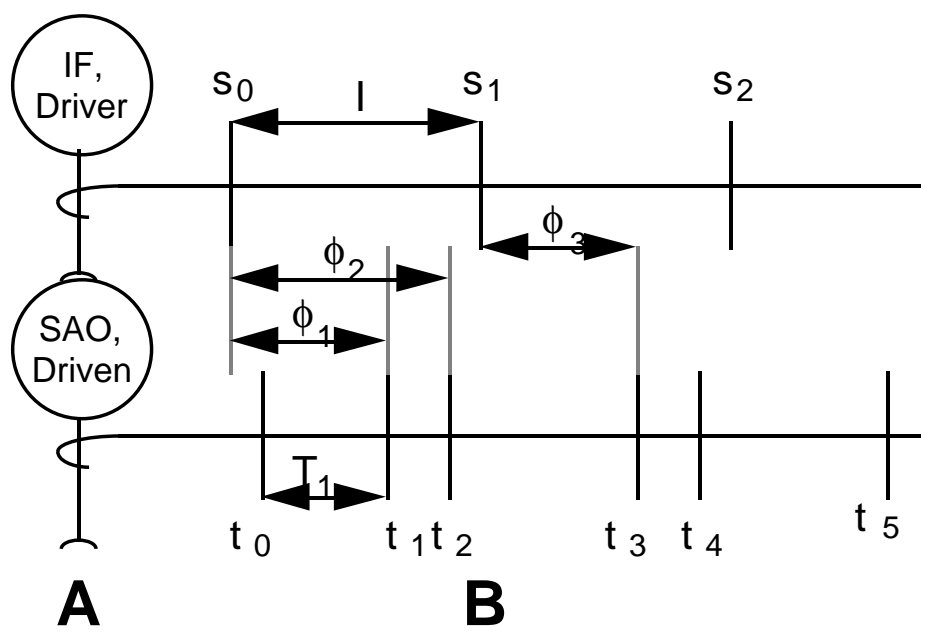

Figure 1: Experimental setup sketch and analysis term deÆnitionÆxperiments were performed on a 2-neuron network (A), with Æringf presynaptic neuron (IF, driver) axon the control variable, and changes in Æringmes of postsynaptic neuron (SAO, driven) recorded. Analysis was performed on intervals between times of occurrence of driver and driven spikes (B).

This paper seeks to frame these questions more precisely using recent results from physiological neural model simulations, data from living preparations, and nonlinear dynamical analysis.

\section{Behaviors in Living and Simulated Neurons}

The living preparation used to serve as our exemplar is the embodiment of a prototypical inhibitory synapse, the crayÆsklowly adapting stretch receptor organ (SAO). The experimental setup, schematized in Figure 1(A), involved a single presynaptic inhibitory Æbeand a single postsynaptic neuron [3]. Because of its prototypical nature, we would expect to see the same responses in any other synapse, at least as a working hypothesis. The model used is based on the physiology of the living preparation and on the the experimental setup; its behaviors have been found to be in close agreement with that of the living preparation [6].

Each neuron produces a series, or train, of spikes. In each train, each spike was identiÆed and attributed an order and a time of occurrence (Figure 1(B)): $k$ and $s_{k}$, respectively, pre- 
synaptically, and $i$ and $t_{i}$ post-synaptically $(k, i=0,1,2, \ldots)$. Each spike was assigned certain intervals: in IF trains, to the $k^{\text {th }}$ spike, the interval $I_{k}$ to the last IF Æringin SAO trains, to the $i^{\text {th }}$ spike, intervals to the last SAO and IF spikes, $T_{i}$ and $\phi_{i}$ (the latter its phase), respectively. The data was thus reduced to the point processes formed by the ordered sets of intervals $I_{k}$ (all identical for pacemaker driving, $I_{k}=I$ ), $T_{i}$ (with mean value $T$ ), and $\phi_{i}[3,7]$.

The postsynaptic, driven neuron is a pacemaker. Its undisturbed, natural discharge was a sequence $T_{i}$ that differed little from its average $T=N$ [3]. The behaviors described here were in response to pacemaker driving, in which the presynaptic spikes were all separated by an essentially invariant interval $I$ [3]. Though analysis focused on resulting stationary postsynaptic discharges, the forms seen in stationary or similar driving are also seen with more complex input regimes [5]. Therefore, we consider such forms as the elementary building blocks of synaptic behavior.

The behavior associated with each inhibitory train was identiÆeds locked, intermittent (including phase walk-throughs), messy (erratic or stammering), or hopping, after those deÆneid nonlinear dynamics [8]. Criteria were based on spike timings of the presynaptic and postsynaptic trains individually and jointly as determined using the data analysis techniques described in [3, $9,10]$.

A locked response is deÆneals a Æxedepeating sequence of $\phi_{i}$ and $T_{i}$, and a behavior was called TMlocked $p: q "$ if these sequences repeated every $q$ SAO spikes $\left(\phi_{i}=\phi_{i+q}\right.$ and $\left.T_{i}=T_{i+q}\right)$ and $p$ IF spikes (so $p I=q T$ ). Locking is a periodic behavior, where the TMinternal state" of the neuron returns to the same value after a period of $q \mathrm{SAO}$ and $p$ IF spikes.

Intermittent is a descriptive term used for behaviors which are almost locked, but are not quite periodic [8]. This includes quasiperiodic behaviors, such as phase slidings and walkthroughs, in which $T / I \approx p / q$. However, in these cases, $T / I$ is an irrational number, and the neuron's state never returns precisely to any previous value. 
The third stationary behavior noted was described as messy, and included both erratic (at low pacemaker driving rates, $I>N$ ) and stammering (at high rates, $I \leq N$ ); the former hypothesized to be the work of deterministic chaos [6, 11], and the latter the action of noise. Erratic discharges have no readily apparent patterning. Stammering, on the other hand, is an example of windowed behavior, where the SAO is able to Ærenly within a narrow interval of time relative to IF spike arrival, so all SAO intervals were essentially multiples of $I$, i.e., $T_{i} \approx k I$, for $k=1,2, \ldots$

Finally, hopping was a situation in which the SAO shifted occasionally from one type of stationary discharge to another. This has been assigned to noise TMbumping" a system among several dynamical attractors.

\subsection{Behaviors Viewed in Terms of Average Rates}

Let us consider the effects of changing the input rate in this preparation and, to simplify matters, we will look at average rates, $1 / I$ and $1 / T$, rather than details. This reduces the observed behaviors to the level of detail seen in ANNs with unit output a scaled version of $1 / I$ or $1 / T$.

Figure 2 schematizes the outputs of an ANN unit with weighted-sum and squashing function (dashed curve) and 1/T for either the SAO or the physiological model (solid lines and dots), as a function of input frequency for inhibitory input. The key features to note are that the TMrealistic" responses consist of paradoxical regions of locking (thick lines), where increasing inhibitory input increases output rate, alternating with non-locked behaviors (dots) [2]. While the overall trend is decreasing, it is only locally monotonic, and those monotonic areas' slopes are opposite the overall trend. In contrast, the output of a typical ANN model is a monotonic and smoothly decreasing. 


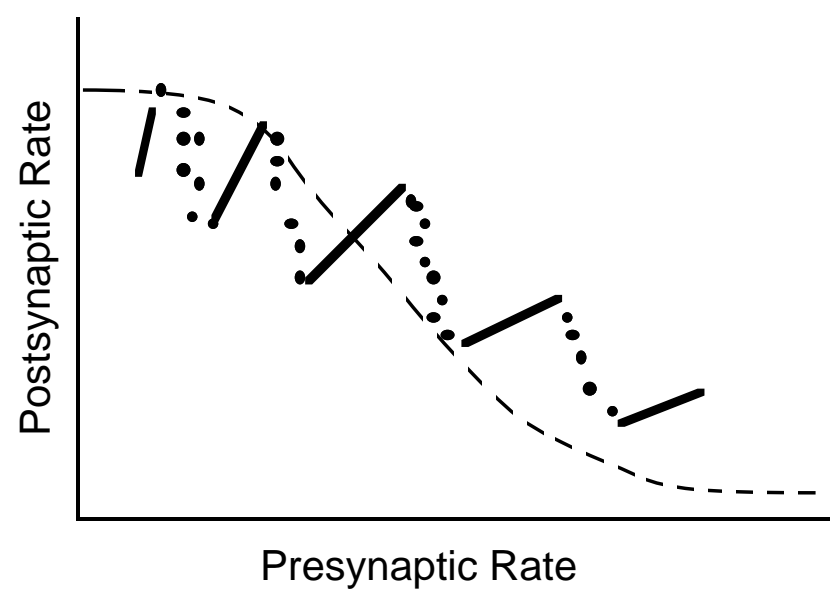

Figure 2: Responses of a dynamical neural model compared to a weighted-sum ANN unit, in response to inhibitory input. For dynamical model, thick lines indicate locking regions, while dots indicate non-locked outputs. ANN unit output is shown by the dashed curve. Figure adapted from [2].

\section{Synaptic Modi/EcationEffects on Behavior}

One advantage of simulation over experiment is that the power of the synaptic coupling can be altered. This change in connectivity can be considered analogous to changing the weight $w$ in a weighted-sum ANN. The resulting behaviors are conveniently summarized by an Arnol'd map [12] or two-dimensional bifurcation diagram, shown in Figure 3, which illustrates the type of output behavior as a function of input frequency (normalized as $N / I$ ) and input amplitude ( $\bar{P}_{\text {syn }}$, the maximum synaptic permeability to $\mathrm{Cl}^{-}$, the inhibitory carrier ion here).

We see in the Ægurthat a locking at any particular ratio (the labeled white areas in the graph) occupies a contiguous, vertically elongated region of the $\left(\bar{P}_{s y n}, I\right)$ plane; hence their usual appellation, tongues. The widths of these tongues vary systematically, according to a Farey series, with the widest tongue between $p: q$ and $p^{\prime}: q^{\prime}$ being $p+p^{\prime}: q+q^{\prime}$ (not readily apparent in the Ægurbecause of the coarse sampling of the parameters). Between these tongues lie parameter regions which produced nonlocked behaviors (cross-hatched areas), including all of those seen in the living preparation. The TMhorizontal slice" at any particular value of $\bar{P}_{s y n}$ 


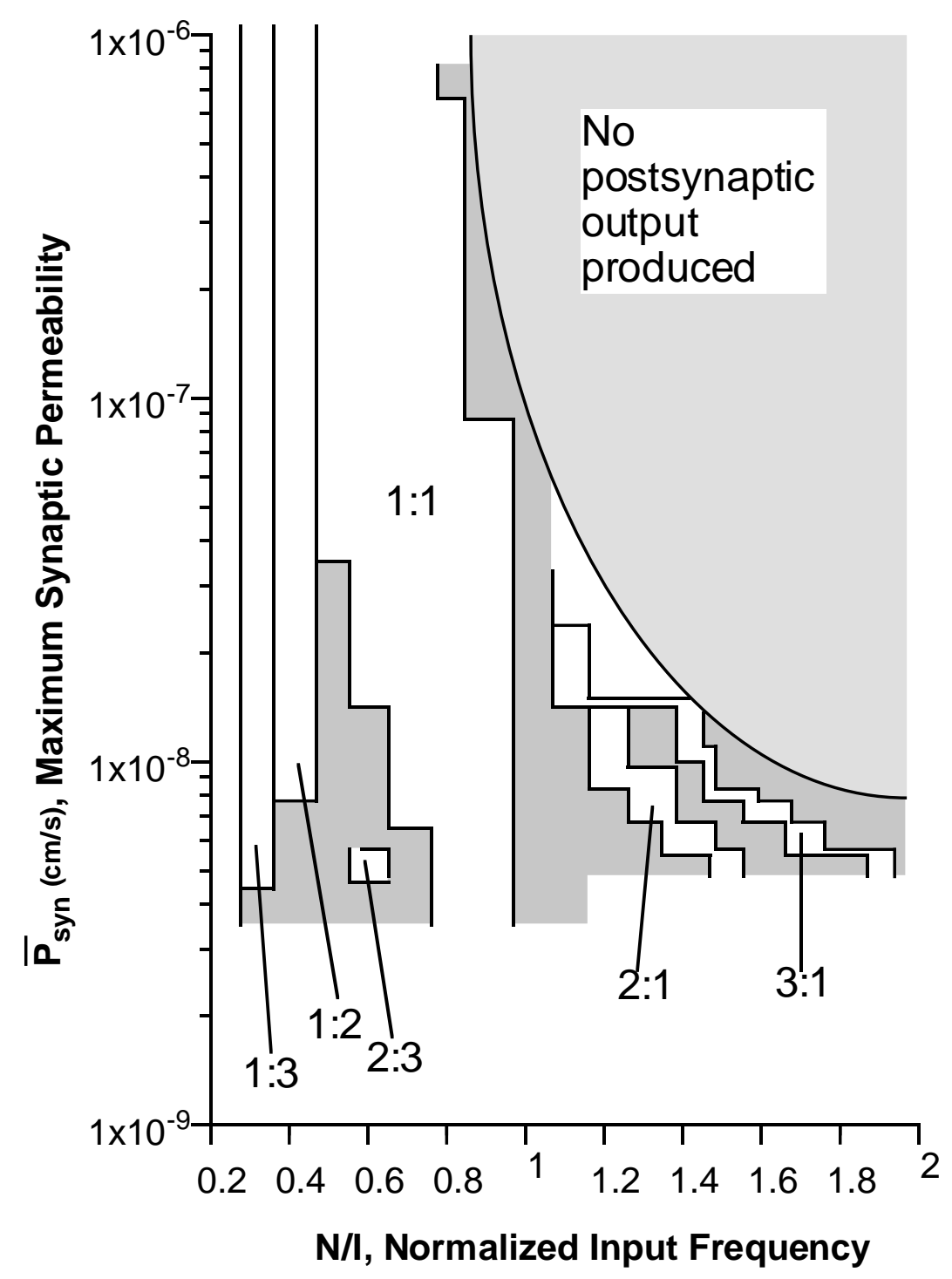

Figure 3: Arnol'd map from simulations with inhibitory input. White areas indicate parameter ranges which produced lockings (ratios noted). Cross-hatched areas are non-locked behaviors.

corresponds to a one-dimensional bifurcation diagram; if we were to plot average postsynaptic rate instead of behavioral category, we would produce a graph similar to Figure 2.

How does changing the synaptic strength change the behavior pattern? The locking tongues extend all the way down to values of $\bar{P}_{\text {syn }}$ that no longer have signiÆcantractical effects on behavior. However, they narrow as $\bar{P}_{\text {syn }}$ decreases. This corresponds to reducing the widths of the paradoxically-sloped line segments in Figure 2. Noting the preponderance of walkthroughs 


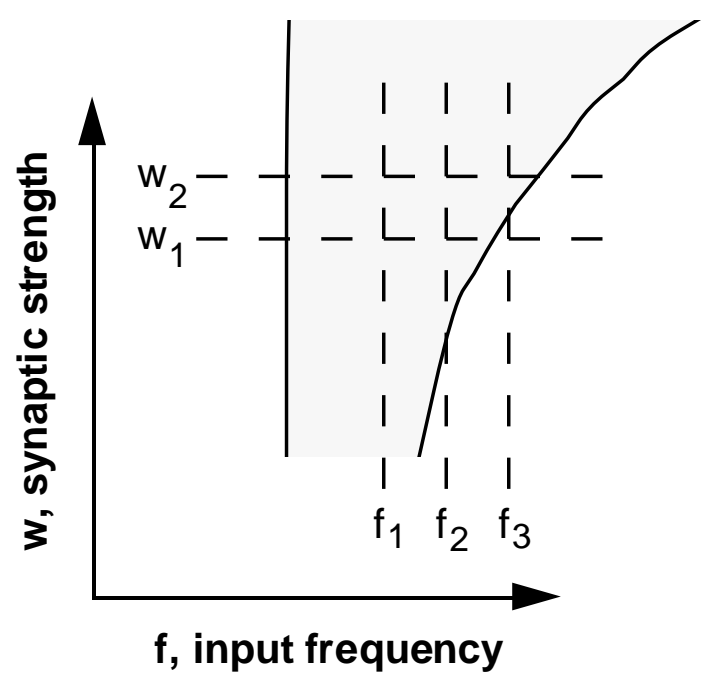

Figure 4: Illustration of effects of changing synaptic strength, $w$, on neural behavior. The shaded area represents part of a locking tongue in an Arnol'd map. A small change in synaptic weight from $w_{1}$ to $w_{2}$ can change a non-locked behavior at $f_{3}$ to a locked one, or have no effect at all, such as at $f_{2}$ or $f_{1}$.

among the nonlocked behaviors at low $\bar{P}_{\text {syn }}[6]$, we see that the overall effect will be to make the synaptic mapping more monotonic, as far as average rates are concerned.

For higher values of $\bar{P}_{s y n}$, we see pronounced locking tongues, amounting to about onethird of the input rate scale in the SAO [4]. Varying $\bar{P}_{s y n}$ within this range can have either no effect or dramatic effect on behavior, as illustrated by the diagram of a locking tongue presented in Figure 4, where the ANN-like terms of $w$ and $f$ have been substituted for $\bar{P}_{s y n}$ and $N / I$, respectively. For presynaptic frequencies $f_{1}$ and $f_{2}$, changing the synaptic strength from $w_{1}$ to $w_{2}$ has no effect on their corresponding postsynaptic rates; this is true for each frequency $f_{j}$ within the tongue at both $w_{1}$ and $w_{2}$. For this range, $\Delta$ (behavior) $/\left.\Delta w\right|_{f=f_{j}}=0$. Conversely, for $f_{3}$, a change from $w_{1}$ to $w_{2}$ induces a change from nonlocked to locked behavior. This can be true even if $w_{2}-w_{1}$ is inÆnitesimallsmall, if $f_{3}$ is close enough to the bifurcation point represented by the tongue boundary. Besides the lack of change in behavior within tongues and the abrupt changes in behavior near tongue boundaries, there are behavior changes among the 
nonlocked behaviors between the tongues, which include walkthroughs and messy behaviors in about equal proportions in the SAO [4].

\section{Conclusions}

Though we cannot state a deÆnitiveynaptic modiÆcationle currently, the above observations can serve to constrain some of its qualities. First of all, it is important to note the complex responses of neurons to inputs with different interspike interval patterns, changes in input frequency, and changes in synapse power. In recurrent ANNs, the effects of dynamics (such as bifurcation behavior) on learning rules must either be eliminated or carefully controlled to achieve the desired results [13]. It is our feeling that a synaptic modiÆcatioprocedure in networks of more realistic elements should take advantage of the individuals' dynamics; greater complexity at that level should lead to greater network computational power. To be fair, however, it could certainly be argued that, in large networks with high interconnectivity, the individual elements' detailed dynamics are washed out.

Based on the previous discussion of coding at small and large synaptic strengths, we can make some general conclusions. With weak coupling, the coding through the synapse is more monotonic, since the locking regions are narrow. The tradeoff for this is that, as the synapse is made weaker, the effect of input at that synapse is reduced. Weak synapses would have to gain a noticeable effect through correlation at multiple sites. However, strict temporal correlation at multiple sites would tend to produce the same types of behaviors as a single strong synapse. Therefore, for monotonic coding through multiple weak connections, steps would need to be taken to reduce synchronization among the inputs, so that the postsynaptic unit would respond to overall input intensity, rather than input pattern.

With higher values of $\bar{P}_{s y n}$ (or multiple correlated inputs), a complex code can arise, whose 
functional signiÆcandis currently unknown. It is important to note, though, that the arrangement of different behaviors is not random; there is a systematic progression from one to another [4]. Potentially, then, this complexity could have useful computational applications. A learning scheme at this level of coupling would essentially be adjusting the proportions of the different behaviors, rather than their locations along the input rate scale (the latter being determined by the neural dynamics itself).

We can conclude that any synaptic modiÆcatioprocess implemented for dynamical, neuronlike elements must take into account: the tradeoff between synaptic monotonicity and efÆcacy synchronization and desynchronization of inputs, and control of behavior proportionality. Exactly how these issues should be dealt with depends on the computational signiÆcancef the different behavior types, a topic of ongoing investigation.

\section{References}

[1] J. Segundo, J.-F. Vibert, M. Stiber, and S. Hanneton, TMSynaptic coding of periodically modulated spike trains," in IEEE International Conference on Neural Networks, 1993.

[2] J. Segundo and D. Perkel, TMThe nerve cell as an analyzer of spike trains," in The Interneuron: UCLA Forum in Medical Sciences (M. Brazier, ed.), (Los Angeles), pp. 349 \pm 89 , University of California Press, 1969.

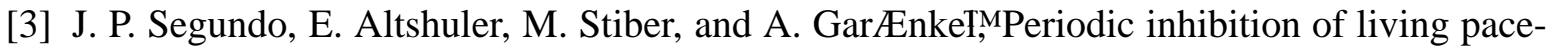
maker neurons: I. Locked, intermittent, messy, and hopping behaviors," Int. J. Bifurcation

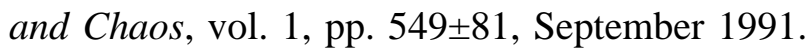

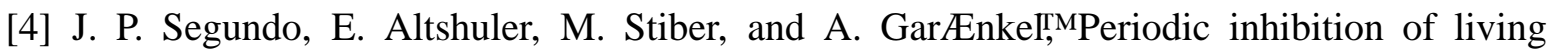
pacemaker neurons: II. InØuence of driver rates and transients and of non-driven post- 


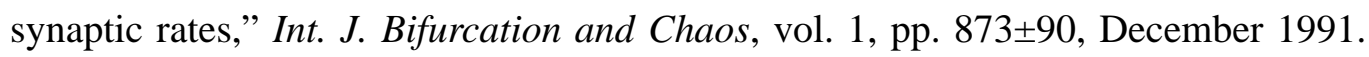

[5] J. Segundo, M. Stiber, E. Altshuler, and J.-F. Vibert, TMTransients in the inhibitory driving of neurons and their post-synaptic consequences," Neuroscience, submitted, 1993.

[6] M. Stiber, Dynamics of Synaptic Integration. $\mathrm{PhD}$ thesis, University of California, Los Angeles, 1992.

[7] D. Cox and P. Lewis, The Statistical Analysis of Series of Events. London: Methuen, 1966.

[8] P. Bergé, Y. Pomeau, and C. Vidal, Order Within Chaos: A Deterministic Approach to Turbulence. New York: Wiley, 1986.

[9] M. Stiber and W. J. Karplus, TMArnol'd map synthesis for periodically forced oscillators," Tech. Rep. HKUST-CS93-5, HKUST Computer Science Department, 1993.

[10] M. Stiber and J. P. Segundo, TMNonlinear dynamics and complex synaptic transfer functions," in IEEE International Conference on Neural Networks, 1993.

[11] G. Sugihara, D. Grace, J. Segundo, and M. Stiber in preparation, 1993.

[12] V. Arnol'd, Geometrical Methods in the Theory of Ordinary Differential Equations. New York: Springer-Verlag, 1983.

[13] K. Doya, TMBifurcations of recurrent neural networks in gradient descent learning," IEEE Transactions on Neural Networks, submitted, 1993. 